\title{
LONG-RANGE RESONANT WAKEFIELDS AT HIGH GROUP VELOCITY*
}

\author{
A.V. Smirnov, D. Yu, DULY Research Inc., Rancho Palos Verdes, CA 90275 \\ E.S. Masunov, MEPhI, Moscow 115409, Russian Federation
}

\begin{abstract}
Synchronous wakefields in a dispersive waveguide are formulated in a general form. The fundamental role of group velocity in wakefield propagation, calculation of attenuation, amplitudes, form-factors and loss-factors is demonstrated for single-bunch and multi-bunch train. Waveguide adiabatic tapering and bunch density variations are taken into account analytically in the timedomain. Application of this approach to oversized pipe with small corrugations and roughness is discussed.
\end{abstract}

\section{INTRODUCTION}

The resonant wakefields induced in a slow wave structure are an important problem of accelerator physics which is associated usually with beam loading and instabilities, rf-generation and energy loss at high order modes (HOMs). The development of novel slow wave structures based on emerging technologies and new materials calls for consideration of the problem under more general assumptions. Generalization is especially important when the group velocity is not small compared to the particle velocity. For future linear colliders the normalized group velocity $\beta_{g r}$ is expected to be about 0.1 [1]. A similar situation takes place in some new rf structures, e.g. in planar mm-wave flat-field structures [2] that can be used for both rf-generation and acceleration. Well-known jungle gym [3] and DAW structures [4] have high group velocity $\beta_{g r}=0.2-0.43$.

An important issue for linear colliders is the development of effective high-frequency rf sources. One of the most promising schemes utilizes the heavy beam loading effect of an intense, relativistic bunched train in a slow wave structure for direct transformation of linac beam power into extracted rf power. Dielectric-loaded waveguide structures $[5,6,7]$ can be very efficient for mmwave power generation with ultrarelativistic beams. Cherenkov radiators of this kind usually have a high group velocity in the range of $(0.1-0.7) c$ [8]. The simplicity of this method makes this technology an attractive option for future high-energy linear colliders. In addition, it is relevant to a number of proposals to use dielectric loaded structures as collinear wakefield accelerators $[9,10]$.

Cherenkov radiation in a waveguide pipe with dielectric [11] and in some slow wave systems [12] was considered on a strictly electro-dynamics basis for point charge exciting monopole mode in an infinitely long uniform waveguide. This paper is a generalized extension of $[11,12]$ for matched slow wave waveguides (including tapered periodic) and a bunch with variable parameters [13]. The approach applied here also includes the effect of dispersion of group velocity causing wave front diffusion that can be important for long dispersive structures or at operating frequencies near the cut-off [13].

\section{SINGLE-BUNCH FIELDS}

We assume here that the resonant frequency $\omega_{1 s}$ is always inside the passband and relatively constant with respect to bandwidth along the matched waveguide. We do not include dispersion of the group velocity $\partial v_{g r s} / \partial h$ and velocity spread in the bunch. The bunch velocity $v\left(z_{q}\right)=d z_{q} / d t$, its transverse position $\vec{\rho}_{\perp}\left(z_{q}\right)$, linear charge density $\eta\left(z, z^{\prime}\right)$, group velocity $v_{g r s}(z)$ and shunt impedance $R_{s}(z)$ are assumed, slowly varying functions of the longitudinal coordinate $z$ in the scale of corresponding wavelength of radiation:

$\frac{\partial}{\partial z} \ln \left\{v(z), \vec{\rho}_{\perp}(z), \eta\left(z, z^{\prime}\right), R_{s}(z), v_{g r s}(z)\right\}<<h_{s}^{\prime}, \quad$ where $h_{s}^{\prime}=2 \pi / \lambda_{s}$ is radiation wave number for the waveguide eigenmodes.

It is convenient to represent the current density of the bunch of finite lifetime $\tau_{0}=\int_{0}^{L} d z / v$ passing through the infinitely long structure:

$$
\vec{j}(\vec{r}, t)=v \vec{e}_{z} \delta\left(\vec{r}_{\perp}-\vec{\rho}_{\perp}\right) \eta\left(z, z^{\prime}\right)\left(\Pi(t)-\Pi\left(t-\tau_{0}\right)\right),
$$

where, $\Pi(t)$ is the Heaviside function, and $\delta\left(\vec{r}_{\perp}\right)=\delta(x) \delta(y)$ is the 2D delta-function.

In the frequency domain we apply a general approach developed by Vainshtein [14] and Solntsev [15]. This approach is based on the field expansion in series of eigenmodes: $\vec{E}_{ \pm s}=\vec{E}_{ \pm s}^{0}\left(z, \vec{r}_{\perp}\right) \exp \left( \pm i \bar{h}_{s} z\right)$ for the forward and backward waves; where

$\bar{h}_{s}=\frac{1}{z} \int_{0}^{z} h_{s} d z=\bar{h}_{s}^{\prime}+i \bar{h}_{s}^{\prime \prime}, h_{s}^{\prime}>0$, and $h_{s}=h_{s}(\omega)$ is the complex wave number. We substitute the Fouriertransform $j_{z}(\vec{r}, \omega)$ of the current $\vec{j}(\vec{r}, t)$ from (1) into the Vainshtein's equations of waveguide excitation written in explicit integral form for the fields. The inverse Fourier

\footnotetext{
* The work is supported by DOE SBIR No. DE-FG03-96ER82213.
} 
transformation with residuals gives, after some algebra, the following expression for electric field of the given mode $s$ :

$$
\vec{E}_{s}(\vec{r}, t)=-2 q \operatorname{Re} F_{s}(z, t) \frac{\vec{E}_{s}^{0}\left(z, \vec{r}_{\perp}(z)\right) E_{-s z}^{0}\left(z_{1}, \vec{\rho}_{\perp}\left(z_{1}\right)\right)}{\left|N_{s}\right| \cdot\left(\bar{v}(z)^{-1}-\bar{v}_{g r s}(z)^{-1}\right)},(2)
$$

where $\Pi(x)$ is symmetric step-function: $\Pi(0)=1 / 2$;

$F_{s}(z, t)=\tilde{\Phi}_{s}\left(z, z_{1}\right) \exp \left(\tilde{\bar{h}}_{s}(z-\bar{v} t)\right)\left[\Pi\left(t_{1}\right)-\Pi\left(t_{1}-\tau_{0}\right)\right] \times$

$\Pi\left((\bar{v} t-z) /\left(\bar{v}-\bar{v}_{g r s}\right)\right) ; \tilde{\bar{h}}_{s}=\bar{h}_{s}^{\prime}-i \bar{h}_{s}^{\prime \prime} \bar{v}_{g r s} /\left(\bar{v}-\bar{v}_{g r s}\right)$;

$N_{s}=\frac{c}{4 \pi} \int_{\perp} d S\left\{\vec{E}_{s} \times \vec{H}_{-s}-\vec{E}_{-s} \times \vec{H}_{s}\right\}$

$z_{1}=\frac{z-\bar{v}_{g r s}(z) t}{1-\bar{v}_{g r s}(z) / \bar{v}(z)} ; t_{1}=\frac{z-\bar{v}_{g r s}(z) t}{\bar{v}(z)-\bar{v}_{g r s}(z)} ;$ and

$\tilde{\Phi}_{s}\left(z, z_{1}\right)=\frac{1}{q} \int_{l\left(z_{1}\right)} d z^{\prime} \eta\left(z_{1}, z^{\prime}\right) \exp \left(-i \tilde{\bar{h}}_{s} z^{\prime} \frac{\bar{v}(z)}{\bar{v}\left(z_{1}\right)}\right)$ is the

generalized bunch formfactor.

Using the longitudinal shunt impedance per unit length $r_{s}(x, y, z)=E_{s z}^{0}\left(z, r_{\perp}\right)^{2} /\left|d P_{s} / d z\right|$ the longitudinal field can be expressed from (2):

$$
\begin{aligned}
& E_{z s}(\vec{r}, t)=-\frac{q}{2} \omega_{1} \operatorname{Re} F_{s}(z, t) \times \\
& \frac{\sqrt{\bar{v}_{g r s} / \bar{v}_{g r s}\left(z_{1}(z, t)\right)}}{1-\bar{v}_{g r s}(z) / \bar{v}(z)} \sqrt{\frac{r_{s}\left(z, \vec{r}_{\perp}\right)}{Q_{s}(z)} \frac{r_{s}\left(z_{1}(z, t), \vec{\rho}_{\perp}\left(z_{1}\right)\right)}{Q_{s}\left(z_{1}(z, t)\right)}},
\end{aligned}
$$

where $Q_{s}$ is the waveguide $Q$-factor for the mode $s$. At the absence of transverse motion the modal loss factor in tapered waveguide follows directly from (3):

$$
k_{q s} \equiv-\frac{1}{q} \int_{-\infty}^{+\infty} E_{z s}(\vec{r}, z / \bar{v}) d z=\frac{\omega_{1}}{4} \int_{0}^{L} \frac{r_{s}\left(z, \vec{\rho}_{\perp}(z)\right) \operatorname{Re} \widetilde{\Phi}_{s}(z) d z}{Q_{s}(z)\left(1-\bar{v}_{g r s}(z) / \bar{v}(z)\right)}
$$

The group velocity effects were included in $[16,17,18]$ as a factor $1 /\left(1-\beta_{g r s}\right)$ for the losses.

Let us discuss the case $1-\beta_{g r s}<<1, \gamma>>1$. Pipes loaded by dielectric or small periodic corrugations have similar properties [19] and can correspond to this case [20]. One can see the value $\left(r_{s} / Q_{s}\right) /\left(1-\beta_{g r s}\right)$ remains finite [20] at $\beta_{g r s} \rightarrow 1$. With the exception of the case of perfect (lowloss) structure, the resonant wakefield becomes short due to high 'dynamic' attenuation $\operatorname{Im} \tilde{\bar{h}}_{s}$ (see $(2,3)$ ). In addition, non-resonant and resistive wakefields can be dominant. Note, resonant wakefield in waveguide relies on the approximation of an infinitely long structure. From the compression effect it follows that structure length should be long enough so that the wakefield is longer than the bunch length: $L\left(v / v_{\text {grs }}-1\right)>>l_{b}$.

For periodic structures an additional relationship will be imposed [21,22]: $N_{p e r}>2 N_{f}$, where $N_{p e r}$ is the number of periods, and $N_{f}$ is the Fresnel number at wavelength $\lambda$ for a single cell. For circular structure with small corrugations we have: $N_{f}=a^{2} / D \lambda \gg 1$, where $D$ is the period and $a$ is the aperture radius. It means that we cannot use the formulae above to estimate wake losses caused by realistic irregular roughness. From the resonance condition at $\beta_{g r s}-1<<1$ it follows that imperfections of periodicity cause considerable frequency detuning which is proportional to $v_{g r} / v$. Other methods based on impedance or diffraction could be more relevant in this case.

\section{BEAM-INDUCED FIELD}

To recall the simple formulae of beam loading when the group velocity is substantial we assume $\left\{v, \Phi, T_{b}, q\right\}=$ const $, \vec{\rho}_{\perp}=0=r_{\perp}, \quad$ and $\quad$ a constant impedance waveguide without detuning: $T_{b}=K \cdot 2 \pi / \omega_{1}$, where $K$ is an integer number of beam subharmonic with respect to the resonant frequency. The field amplitude $E_{A s}$ is defined here as:

$$
E_{z}(r, t)=\operatorname{Re} E_{A s}(z, t) \exp \left(i\left(h_{s}^{\prime} z-\omega_{1} t\right)\right) .
$$

The field in the transient regime can be obtained using the principle of superposition applied to (3):

$E_{A s}=-I r_{s} \widetilde{\Phi}_{s}\left(1-\exp \left(\omega_{s}^{\prime \prime}(z / v-t) /\left(1-v_{g r s} / v\right)\right)\right) \Pi(t-z / v)$, , (5)

where $I=q / T_{b}$ is the average beam current, $t<z / v_{\text {grs }}$, if $v_{\text {grs }}>0 ; t<L / v+(L-z) /\left|v_{g r s}\right|$, if $v_{g r s}<0$.

In the steady-state regime, when $t>z / v_{g r s}$, if $v_{g r s}>0$; and $t>L / v+(L-z) /\left|v_{g r s}\right|$, if $\quad v_{g r s}<0$ expression (3) gives exactly the same result known from the standard formulation $[12,3]$ :

$$
E_{A s}=-I r_{s} \tilde{\Phi}_{s}\left[1-\exp \left(\begin{array}{c}
-h_{s}^{\prime \prime} z, \text { if } v_{g r s}>0 \\
h_{s}^{\prime \prime}(L-z), \text { if } v_{g r s}<0
\end{array}\right)\right] .
$$

Hence, the average power generated by the steady-state beam loading at high group velocity satisfies the same formula known from the conventional approach:

$$
P_{s t}=\left.\frac{\left|E_{s z}\right|^{2}}{2 h_{s}^{\prime \prime} r_{s}} \approx\left|I \Phi_{s} L\right|^{2} \frac{\omega}{4} \frac{r_{s}}{Q_{s}} \frac{1}{\left|v_{g r s}\right|}\right|_{s} ^{\prime \prime} L \ll<1 .
$$

It is important to note, non-small group velocity (compared to the bunch velocity) can induce pulsation in output power. The generalized conditions for slowlyvarying amplitude (the fields are long-range) follows from (2):

$$
L\left|\bar{v}_{g r s}^{-1}(L)-\bar{v}^{-1}(L)\right|>>T_{b} ; \quad 2 Q_{s}\left(v-v_{g r s}\right)>>c .
$$

If the amplitude is slowly-varying and (8) is satisfied, one can apply to (2) the superposition principle in an integral form. In more general form the long-range fields (including HOMs) can be represented in terms of the dimensionless amplitude $C_{s}(z, t)$ :

$$
\frac{\partial C_{s}}{\partial t}+v_{g r s} \frac{\partial C_{s}}{d z}+i\left(\omega_{1 s}^{\prime}-\omega\right) C_{s}=\frac{2 v_{g r s}}{N_{s}} \int_{\perp} d S \overline{\vec{j}}_{\omega} \vec{E}_{-s}\left(\vec{r}_{\perp}\right),(9)
$$

where $\vec{E}(\vec{r}, t)=\operatorname{Re} C_{s}(z, t) \vec{E}_{s}(\vec{r}) \exp (-i \omega t), \quad \omega$ is the characteristic frequency of the interaction process (e.g. $2 \pi / T_{b}$ for beam loading or instability frequency for BBU 
effect), $\overline{\bar{j}}_{\omega}=\frac{\omega}{2 \pi} \int_{t-2 \pi / \omega}^{t} \vec{j}(\vec{r}, \tau) e^{i \omega \tau} d \tau$. The initial/boundary conditions are trivial for steady-state regime: $\left.C_{s}(0, t)\right|_{v_{g r}>0}=0,\left.C_{s}(L, t)\right|_{v_{g r}<0}=0$. However, for the transient regime we have now: $C_{s}(z, t<z / \bar{v})=0$. Equation (9) is well-known in TWT/BWO theory [23] and can be derived also from other models without imposing limitations on $v_{g r} / v$ value. Those include models of coupled cavities and the model of the cavity excitation by summation over modes having different longitudinal wavenumbers [13].

\section{CONCLUSION}

If the group velocity is not small compared to the bunch velocity, it can play an important role in the magnitude of resonant fields induced by single bunch and in beam loading in the transient regime. For example, in a linac with low-energy injection and low-gain tapered buncher section, the $v_{g r} / v$ ratio can be as high as 0.57 [24]. Therefore, the group velocity effect will have a noticeable impact on the wakefields and beam dynamics especially for the high-current, stored-energy mode of linac operation [13]. It was confirmed numerically for strongly tapered bunching/accelerating sections. Retarded arguments and the $1 /\left(1-v_{g l} / v\right)$ factor were included in these self-consistent simulations [25,26] performed for transient beam loading. This effect should be taken into account in designing a linear collider operating in transient regime or in a single-bunch mode. The accuracy of short-range field calculations based on the modal loss-factors can be improved by including the group velocity effect in the loss-factor computations.

BBU simulations for Drive Beam Deceleration schemes or for future linear colliders can be influenced by field 'compression/expansion' and group delays in arguments (especially for tapered sections). It can be important in collinear wakefield accelerators as well.

In oversized pipe with small corrugations and finite conductance the resonant wakefields become short, and resistive wakefields can be dominant.

\section{REFERENCES}

[1] P. Chen, J. Irwin, T.W. Markiewicz et al, in Proc. of the DPF/DPB Summer Study on New Directions for HighEnergy Physics, Snowmass, 1996, p. 360.

[2] D. U. L. Yu and A. V. Smirnov, in AIP Conf. Proc. AIP 569 (2000); this Conf., FPAH076

[3] P.B. Wilson, SLAC-PUB 2884 (1981).

[4] V.G. Andreev, J. Tech. Phys., v. 11, N4 (1971)788.

[5] R. Keinigs, M. Jones, and W. Gai, Part. Accel. 24, 223 (1989).

[6] E. Chjonacki, W. Gai, P. Schoessow, and J. Simpson, in Proc. of PAC'91 Conf., IEEE, San Francisco, (1991) 2557.
[7] W. Gai, P. Schoessow, Design and Simulation of a High Frequency High Power RF Extraction Device Using a Dielectric-Loaded Waveguide, to be published in Nuclear Instrum. Meth., A.

[8] W. Gai, Calculation of PETS Using Dielectrics for CLIC type TBA Applications, WF-Note-186, 1999.

[9] R. Keinigs, M. Jones, and W. Gai, Part. Accel. 24, 223 (1989)

[10] T. B. Zhang, J. Hirshfield, T. Marshall, and N. Hafizi, Phys. Rev. E 56, 4647 (1997)

[11] E. S. Masunov, in Izvestiya VUZov, Radiophysika, V. 12, N 10, (1969) 1566.

[12] E.L. Burshtein, G.V. Voskresenskii, "Linear Electron Accelerators with Intense Beams," Atomizdat, Moscow, USSR (1970).

[13] A. V. Smirnov, Ph. D. dissertation, MEPhI (1985).

[14] L. A. Vainshtein, "Electromagnetic Waves," Izd. Sov. Radio, USSR, Moscow, (1957).

[15] L. A. Vainshtein, V.A. Solntsev, "Lectures on RF Electronics," Izd. "Sov. Radio," Moscow (1973).

[16] A. Millich, L. Thorndahl. CLIC Note 366.

[17] E. Chjonacki, R. Conecny, M. Rosing, J. Simpson, in Proc. of PAC'93 Conf., p. 815, 1993; W. Gai, private communications.

[18] H. Braun, M. Valentini, CERN CTF-Note 99/05.

[19] G.V. Voskresenskii, "Nauchnye trudy RAIAN," v.3, N3 (1961) 74 (in Russian)

[20] K.L.F. Bane, G. Stupakov, SLAC-PUB-8599 (2000).

[21] S. A. Heifets, S. A. Kheifets, SLAC-PUB-4625 (1988).

[22] A.V. Smirnov, in Proc. of PAC'99 Conf., NY, 1999 March 29th - April 2nd, p. 3615

[23] V.N. Shevchik, D.I. Trubetskov. "BWO Tube Theory," Saratov, 1975 (in Russian).

[24] A.V. Smirnov, in Proc. of the PAC'95 and Int. Conf., Vol. 2, IEEE AIP (1996) 1152.

[25] A.V. Smirnov, in Voprosy Atomnoy Nauki i Techniki. Seriya: Yaderno-Phizicheskie Issledovaniya. Moscow, N 5(13), 1990, p. 23

[26] A.V. Smirnov, in "Accelerator Physics and Techniques," Moscow, Energoatomizdat, (1985) p.39-44 (in Russian). 